$\S=-1$

\title{
Power Tracing Monitoring incorporating Optimal Reactive Power Dispatch
}

\author{
Nabil Fikri Ruslan ${ }^{\text {a }}$, Ismail Musirin ${ }^{1 a}$, Mohamad Khairuzzaman Mohamad Zamani ${ }^{2 a}$, \\ Muhammad Murtadha Othman ${ }^{3, a}$, Zulkiffli Abdul Hamid ${ }^{4 a}$, Zikri Abadi Baharuddin ${ }^{5 b}$, Nor Azura Md Ghani ${ }^{6 c}$ \\ ${ }^{a}$ Power System Operation Computational Intelligence Research Group (POSC), \\ Faculty of Electrical Engineering, Universiti Teknologi MARA, 40450, Shah Alam, Selangor, Malaysia \\ ${ }^{b}$ Faculty of Information and Communication Technology, University Teknikal Malaysia Melaka (UTeM) \\ ${ }^{c}$ Center for Statistical Studies and Decision Sciences, Faculty of Computer and Mathematical Sciences, Universiti Teknologi MARA, \\ 40450 Shah Alam, Selangor, Malaysia \\ *Corresponding author E-mail: ismailbm@salam.uitm.edu.my
}

\begin{abstract}
General power flow studies do not manage to trace the contributors by generators on power losses in the whole power transmission system. Thus, power tracing approach is utilized to address this issue. Power tracing is a termed used to describe the contribut ors for the power losses dissipated on the transmission line. The traditional technique made use the knowledge of circuit analysis such as cut set theory. However, there was no element of optimization which can help to achieve the optimal solution. This paper presents the power tracing monitoring during voltage stability improvement process, implemented by optimal reactive power dispatch. In this study, the impact of power tracing on voltage stability variation was investigated. Evolutionary Programming (EP) was de veloped and utilized to incorporate power tracing, along with voltage stability improvement. A pre-developed scalar voltage stability index was incorporated to indicate the voltage stability condition. On the other hand, the voltage stability initiative was conducted via the optimal reactive power dispatch. The power tracing was monitored for both; the pre-optimization and post-optimization scenarios. Small system model was tested to realize the power tracing phenomenon, which is rather rare study in power system community. Results on power tracing obtained during the pre- and post-optimal reactive power dispatch revealed that not all generators will involve in the contribution on the total transmission loss in the system. This can be beneficial to power system operators for allocating the cost without discrimination in the long run.
\end{abstract}

\section{Introduction}

Based on definition, power tracing or electricity tracing is a technique used for solving electricity deregulation problem which considers the transmission service pricing. Power tracing consist of two task which are generation tracing and load tracing. First concept has been introduced by Kirschen et al in 1998 [1]. In his works, power tracing concept was developed based on matrix operation concept. [1] He had explained a transmission loss allocation scheme based on the concept of power flow tracking and also proportionality assumption is analysed in terms of the cooperative game theory. [2] In this study, power tracing is solved by using evolutionary programming which are more reliable and shows different ways in performing power tracing process. This technique will show simple and different technique in performing power tracing without any negative sharing and not depending on any assumptions and matrix property which can be applied in any condition. Furthermore, the proposed technique used an artificial intelligence optimization process to provide fair result that validated through several test system performed. This technique has been developed using artificial intelligence optimization technique. The impact of power tracing to the voltage stability and how the improvement of voltage stability affecting power tracing also have been reported in this paper [3]. Power tracing has been an important issue in solving power flow problems. The existing power tracing technique are still not good enough for any assumption and used in any operation where limited its usage especially when it need to be inverse not singularly [4]. While ensure that the energy generated in right amount, the system need to make sure that the energy is generated within feasible cost and maximum power generation [6]. Thus, a method that can be accurately determined the solution to the transmission line problems while satisfy the constraints has been developed [7]. Different from previous methods of power tracing technique, the proposed technique performs generator selection-based $\mathrm{n}$ stability index contributes by each generator in the systems [5]. This paper has presented the power tracing technique for voltage stability for voltage stability improvement using evolutionary programming. In this study, power tracing is identified with regards to voltage stability issue. This study had been implemented on 6-bus systems to demonstrate the voltage stability improvement to be done.

\section{Methods and Material}

Power tracing can be divided into two categories which are generation tracing (GT) and load tracing (LT).

a) Generation Tracing Concept (GT): Tracing power that contributed by the individual generator is called as generation 
tracing. This concept is necessary as performing the tracing process. Theoretically, this concept refers to the line that brings outflow from generators to the load bus is considered generator's dominion.

b) Load Tracing Concept (LT): This concept is vice versa as stated in generation tracing which tracing the power extracted by individual load. As contrast to GT, the line that bring inflow to load bus that connected to those lines are included as load's dominion.

\subsection{Evolutionary Programming}

In this paper, there are three cases which will be included. The first case is focusing on developing on power tracing technique. As for the second case, this paper explains the relationship of power tracing technique whereas the third case is focusing on power tracing and voltage stability improvement. From 6-bus system, the total power loss is $4.225 \mathrm{MW}$.

\section{a) Pre-optimization process}

Figure 1 illustrates the flow of pre-optimization process. This process is the first part of the optimization process. This is the process where the value of reactive power will be increased step by step with an increment of 10 and evaluated based on the normal load flow process. This process is evaluated as in steps below: -

1. Take the first value of 10 MVAR into the system.

2. Take the value and run it under load flow process

3. Then, the result from the load process is calculated on the FVSI algorithm [8].

4. After that, the value of FVSI will be check based on the constraint as the value must not exceed 1 . If the value of FVSI is exceeding 1 , the system will show error.

5. If the value of FVSI is accepted, then it will undergo upstream algorithm to evaluate the generation contribution in the system.

6. Next, it will run under downstream algorithm to evaluate the load extraction in the system.

7. Then, the system losses will be calculated, and the result will be display.

8. Then, the system will be check whether the value of $Q_{d}$ is exceed the required limit which is $60 \mathrm{MVAR}$.

9. If the value of $Q_{d}$ is exceed the maximum value, then the system will be ended. If the value still not exceed maximum value, then the system will increase the value by 10 and the system will repeat from step 2 until the value of $Q_{d}$ is satisfied.

10. Then, the result will be shown in the command window and the value can be tabulated.

\section{b) Optimization process}

Initialization: It is used to generate set of random number represent the variables that control the objective function. This random number must follow the constraint. Then, this random numbers will undergo load flow process and check based on the constraint. This random number can be generated in 1 population or 20 population at one time and in this case, the system will generate 20 random number that satisfy the constraint. This set of random number will be called as parents. The random number is generated using equation (1).

$$
x=\operatorname{rand}(a, b) * 500
$$

where:

$$
a=\text { number of row }
$$

$b=$ number of column
Fitness calculation: Fitness calculation is the equation, function or subroutine that will be used to optimize the random data earlier. It can be a single mathematical equation or long subroutine. It will make sure that the system need to be minimized or maximized.

Mutation: This step is used to breed the offspring. The set of random number which also called as parents will be mutated and produce 20 new number which called as offspring. The mutation process is used based on the Gaussian mutation technique that shown in the equation 2 below.

$$
\begin{aligned}
& x_{i j, \text { new }}=x_{i j}+N\left(\mu, \sigma^{2}\right) \\
& \mu=0 \\
& \sigma^{2}=0, \beta\left(x_{j, \max }-x_{j, \min }\right)\left(\frac{f_{i}}{f_{\max }}\right)
\end{aligned}
$$

Where:

$$
\begin{array}{ll}
x_{i j, n e w}= & \text { mutated parents (offspring) } \\
x_{i j}= & \text { parents } \\
N & =\text { Gaussian distributed random number with mean } \mu \text { and } \\
& \text { variance } \sigma^{2} \\
\beta & =\text { mutation scale, } 0<\beta<1 \\
f_{i}= & \text { fitness value } \\
f_{\max }= & \text { maximum fitness }
\end{array}
$$

Combination: This step is used to combine both parents and offspring in series. Thus, the total set of number will be double and form a set of 40 numbers. The first 20 will be the parents and the second 20 will be the offspring. The command shows how the data been combined.

output_all=[output $1 ;$ output 2$]$

Selection: This step will be selected the survival that suitable for the system to be used later. First, the data that had been combined will be sorts either ascending or descending order. It depends on the objective of the systems. If the system need to be maximized, then the data will be sort in descending order and vice versa. The command below is an example for minimizing the fitness numbers which been used in this project.

rank_op=sortrows(output_all,3)

Convergence test: This step is the last step in the postoptimization process. This step will determine the stopping criterion that will be need in the system later. It is defined based on the minimum fitness and the maximum fitness. Then, the system will be loop back for the next iteration until the set of number has been optimized to its fit value. This test can be done as shown in the command below.

if (max_fitness-min_fitness) $<=0.00001$;

After all these steps had been done, then the finest value will be used and insert to the system that as same as in pre-optimization process, but the result will be different. The result will be slightly lower that the result in pre-optimization process and the gap between the FVSI value in both result will be increased since the system will improve the index value. 


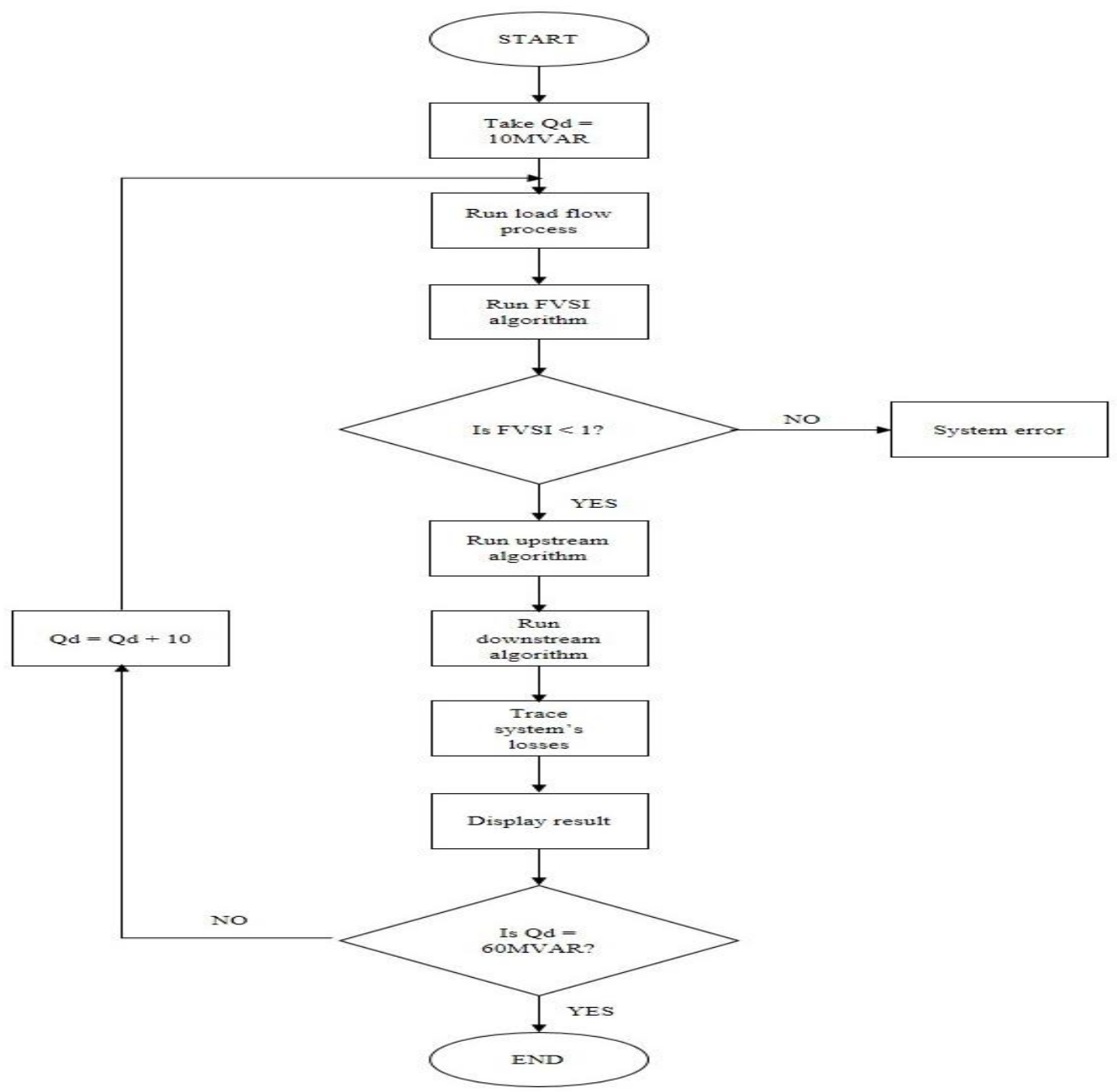

Fig. 1: Flow chart of pre-optimization process

\section{Results and Discussions}

The developed technique was tested on the IEEE 6-bus test system. The result for minimizing the value of FVSI will be explained on the next chapter. The IEEE 6-bus test system is shown on Figure 2 below. This bus system has 2 generator buses (bus 1 and bus 2) and 4 load buses (bus 3 , bus 4 , bus 5 , and bus 6 ). In this project, it consists of 3 parts of experiment. The first one is power tracing to the base case of IEEE 6-bus systems as there are no increment in optimal reactive power dispatch (ORPD). The second is power on the systems with the increment of reactive power dispatch (ORPD) and third is the power tracing concept to the voltage stability improvement.

\subsection{A) Power Tracing for Base System}

As the simulation been ran, total power generated from generators is $102.225 \mathrm{MW}$ and $32.848 \mathrm{MVAR}$. Not only that, it has been found that losses on the line flow is $4.225 \mathrm{MW}$ and 17.848 MVAR. As for the FVSI, the maximum value is 0.1686 which voltage at line from bus 2 to bus 5 .

From Table 1, the generator from bus 1 contributes its power to the load at bus 3 , bus 4 , bus 5 and bus 6 for $12.02 \mathrm{MW}, 10.33 \mathrm{MW}$, $8.64 \mathrm{MW}$ and $31.23 \mathrm{MW}$ respectively. Thus, total generation produced by generator 1 is $62.23 \mathrm{MW}$. As for generator 2 , it only contributes its power generated to the load at bus 3 and bus 5 only which $27.44 \mathrm{MW}$ and $12.56 \mathrm{MW}$. The possible reason is that bus 4 and 6 has received enough power from generator 1, so that it does not need any power contributes by generator 2 . As for downstream algorithms, Table 3 and Table 4 is tabulated for the result of power flow tracing of downstream algorithm.

Table 1: Power Flow Tracing of Upstream Algorithm (Generation Share Contribution to Loads)

\begin{tabular}{|c|c|c|c|}
\hline Load bus & G1 & G2 & Total \\
\hline 3 & 12.0247 & 27.4395 & 39.4642 \\
\hline 4 & 10.3253 & 0.0000 & 10.3252 \\
\hline 5 & 8.6431 & 12.5605 & 21.2036 \\
\hline 6 & 31.2321 & 0.0000 & 31.2321 \\
\hline Total & 62.2251 & 40.0000 & 102.2251 \\
\hline
\end{tabular}

Table 2: Power Flow Tracing of Upstream Algorithm (Generation Share Contribution to Line Flows)

\begin{tabular}{|c|c|c|c|c|}
\hline From & To & G1 & G2 & Total \\
\hline 1 & 6 & 35.7770 & 0.0000 & 35.7770 \\
\hline 1 & 4 & 26.4481 & 0.0000 & 26.4481 \\
\hline 2 & 5 & 0.0000 & 12.5605 & 12.5605 \\
\hline 2 & 3 & 0.0000 & 27.4395 & 27.4395 \\
\hline 4 & 6 & 4.0982 & 0.0000 & 4.0982 \\
\hline 4 & 3 & 12.0247 & 0.0000 & 12.0247 \\
\hline 6 & 5 & 8.6431 & 0.0000 & 8.6431 \\
\hline
\end{tabular}




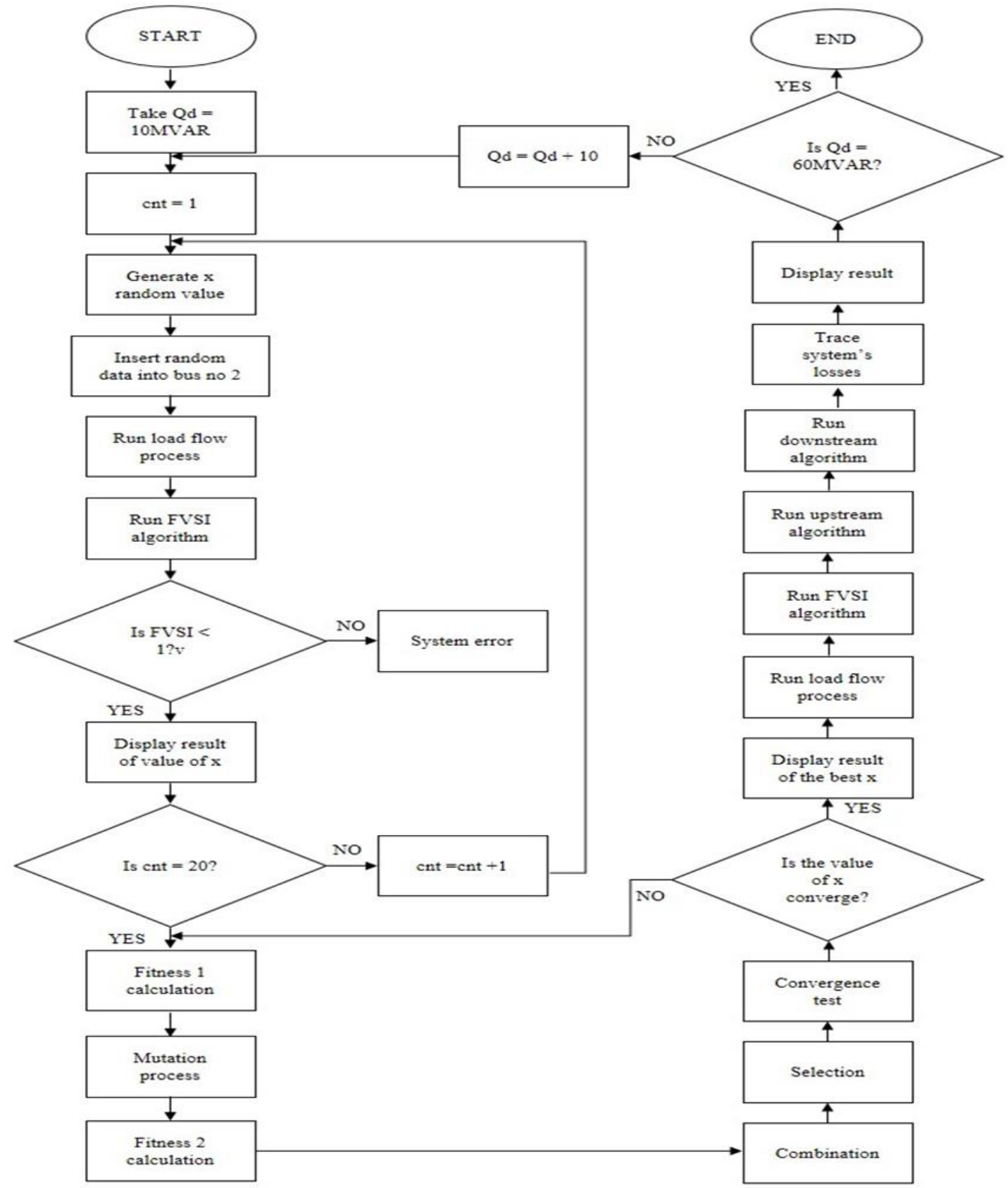

Fig. 2: The flow of optimization process

Table 3: Power Flow Tracing of Downstream Algorithm (Extraction from Generation by Loads)

\begin{tabular}{|c|c|c|c|c|c|}
\hline Generator & Bus 3 & Bus 4 & Bus 5 & Bus 6 & Total \\
\hline G1 & 11.52 & 10.00 & 8.13 & 30.00 & 59.62 \\
\hline G2 & 26.48 & 0.00 & 11.87 & -0.00 & 38.35 \\
\hline Total & 38.00 & 10.00 & 20.00 & 30.00 & 98.00 \\
\hline
\end{tabular}

This algorithm is the same as Upstream Algorithm but has the difference in terms of the way the values are taken as the values taken from the load consumptions. From table 3, it can be found that all the load is consuming the power at their total power demand. The important part that that had to be discussed is the value of power flow at the lines. If the power flow on the line from bus 1 to bus 6 , it can be seen that both value of power in the Upstream and Downstream algorithms are slightly different. In the upstream algorithm, the value of power flow is $35.78 \mathrm{MW}$ while the power flow in the downstream algorithm is $34.20 \mathrm{MW}$. The different of power in both algorithm is $1.57 \mathrm{MW}$. Thus, it can be found that the percentage of losses from bus 1 to bus 6 is about 4.6 percent. Not only this connection, there are losses is all of the line that are exist in the IEEE 6-bus test system. The value of losses which will exist in the transmission lines can be found in Table 5 below.

\subsection{Power Tracing for Pre-Optimization and Post- Optimization Process.}

In this section, the system will be observed in different way. Since the value of reactive power dispatch is inserted manually, the value of FVSI and power flow are observed manually too which can make the output value can be compared easily. Then, there will be the value of $\mathrm{x}$ that generated randomly will be inserted to bus number 2. This value of $\mathrm{x}$ is the value that will be optimized so that the output can be minimized. Table 6 is tabulated to show the comparison of FVSI before and after optimization. The preoptimization process refers to the conventional load flow solution, while the post-optimization refers to the implementation of reactive dispatch to improve voltage stability.

Table 4: Power Flow Tracing of Downstream (Extraction from Line flow by Loads)

\begin{tabular}{|c|c|c|c|c|c|c|}
\hline From & To & Bus 3 & Bus 4 & Bus 5 & Bus 6 & Total \\
\hline 3 & 2 & 26.48 & 0.00 & -0.00 & -0.00 & 26.48 \\
\hline 3 & 4 & 11.52 & 0.00 & -0.00 & -0.00 & 11.52 \\
\hline 4 & 1 & 11.52 & 10.00 & 0.84 & 3.09 & 25.45 \\
\hline 5 & 6 & 0.00 & 0.00 & 8.13 & -0.00 & 8.13 \\
\hline
\end{tabular}




\begin{tabular}{|c|c|c|c|c|c|c|}
\hline 5 & 2 & 0.00 & 0.00 & 11.87 & -0.00 & 11.87 \\
\hline 6 & 1 & 0.00 & 0.00 & 7.29 & 26.91 & 34.20 \\
\hline 6 & 4 & 0.00 & 0.00 & 0.83 & 3.09 & 3.93 \\
\hline
\end{tabular}

Table 5: Tracing of System (MW - Losses)

\begin{tabular}{|c|c|c|c|}
\hline Load Bus & G1 & G2 & Total \\
\hline 3 & 0.5014 & 0.9629 & 1.4642 \\
\hline 4 & 0.3252 & 0.0000 & 0.3252 \\
\hline 5 & 0.5132 & 0.6903 & 1.2036 \\
\hline 6 & 1.2321 & 0.0000 & 1.2321 \\
\hline Total & 2.5719 & 1.6532 & 4.2251 \\
\hline
\end{tabular}

Table 6: Comparison of FVSI before and after optimization process

\begin{tabular}{|c|c|c|c|}
\hline \multirow{2}{*}{$\mathrm{Q}_{\mathrm{d}}$} & \multirow{2}{*}{$\mathrm{Q}_{\text {injected }}$} & \multicolumn{2}{|c|}{ FVSI } \\
\cline { 3 - 4 } & 17.2764 & Before & After \\
\hline 10 & 22.5666 & 0.1894 & 0.1871 \\
\hline 20 & 28.8615 & 0.3484 & 0.2374 \\
\hline 30 & 36.3398 & 0.4439 & 0.3107 \\
\hline 40 & 0.5392 & 0.3860 \\
\hline 50 & 42.7579 & 0.53936 \\
\hline 60 & 45.9824 & 0.6343 & 0.5811 \\
\hline
\end{tabular}

From Figure 3, the graph shows the comparison between preoptimization and post-optimization. As the value of reactive power increases, the value of FVSI will also increase. It will increase until it is reached its unstable condition as it is approaching 1. From the graph, the solid line represents the pre-optimization process while the dotted line represents the post optimization process. It can be seen that the value of FVSI has improved by the implementation of optimal reactive power dispatch.

As for the power flow tracing, the result can be seen in the Appendixes. There will be comparison in upstream and downstream algorithm that will show the difference in power flow before and after optimization. Not only that, the value of reactive power equal to 10MVAR is taken as the reference in comparison to the line flow before and after optimization. Tables 7 and 8 tabulate the value of power losses of the system before and after optimization process. It can be seen the value of losses are decreasing since only generator 1 is supplying the power to the whole system.

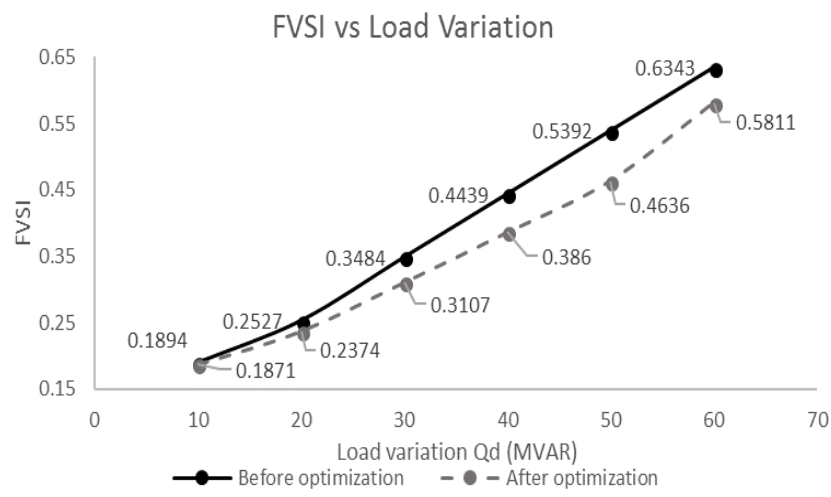

Fig. 3: Comparison of FVSI before and after optimization.

Table 7: Tracing of System Loss (before optimization)

\begin{tabular}{|c|c|c|c|}
\hline Load Bus & G1 & G2 & Total \\
\hline 3 & 0.5542 & 1.0198 & 1.5740 \\
\hline 4 & 0.3610 & 0.0000 & 0.3610 \\
\hline 5 & 0.5289 & 0.7070 & 1.2359 \\
\hline 6 & 1.2882 & 0.0000 & 1.2882 \\
\hline
\end{tabular}

Table 8: Tracing of System Loss (after optimization)

\begin{tabular}{|c|c|c|}
\hline Load Bus & G1 & Total \\
\hline 2 & 0.0000 & 0.0000 \\
\hline 3 & 0.5551 & 0.5551 \\
\hline 4 & 0.3621 & 0.3621 \\
\hline 5 & 0.5305 & 0.5305 \\
\hline 6 & 1.2904 & 1.2904 \\
\hline Total & 2.7380 & 2.7380 \\
\hline
\end{tabular}

\section{Conclusion}

This paper has presented the implementation of evolutionary programming for improving the voltage stability index with power tracing being monitored in the system. This technique has been tested on the IEEE 6 bus test system. Throughout this project, it has been concluded that this technique has working well in order to determine the optimal reactive power dispatch for solving the voltage stability problems. The variation of value of ORPD has been recorded when this value has been inserted into the chosen bus.

\section{Acknowledgement}

The authors would like to acknowledge the Institute of Research Management and Innovation (IRMI) UiTM Shah Alam, Selangor, Malaysia for the financial support of this research. This research is supported by IRMI under the LESTARI Research Grant Scheme with project code: 600-IRMI/DANA 5/3/LESTARI (0117/2016).

\section{References}

[1] Khan B \& Agnihotri G. "A comprehensive review of embedded transmission pricing methods based on power flow tracing techniques", Chinese Journal of Engineering. Vol. 2013, (2013), pp. 113.

[2] Kirschen D, Allan R \& Strbac G. "Contributions of individual generators to loads and flows", IEEE Transactions on Power Systems. Vol. 12, No. 1, (1997), pp. 52-60.

[3] Abdul Hamid Z, Musirin I, Othman MM \& Rahim NA. "Efficient power scheduling via stability index based tracing technique and blended crossover continuous Ant Colony Optimization", Australian Journal of Basic and Applied Sciences. Vol. 5, No. 9, (2011), pp. 1335-1347.

[4] Abdul Hamid Z, Musirin I, Othman MM \& Rahim MNA. "A novel technique for generation tracing via Evolutionary Programming", 2011 5th International Power Engineering and Optimization Conference. (2011), pp. 381-386.

[5] Kalil MR, Musirin I \& Othman MM. "Ant colony based optimization technique for voltage stability control", Proceeding of the WSEAS international conference on power system. (2002), pp. 149154.

[6] Rahmat NA \& Musirin I. "Differential Evolution Ant Colony Optimization (DEACO) technique in solving Economic Load Dispatch problem", 2012 IEEE International Power Engineering and Optimization Conference Melaka, Malaysia. (2012), pp. 263-268.

[7] Abdelkader SM. "Transmission loss allocation through complex power flow tracing", IEEE Transactions on Power Systems. Vol. 22 No. 4, (2007), pp. 2240-2248.

[8] Musirin I \& Abdul Rahman TK. "Novel fast voltage stability index (FVSI) for voltage stability analysis in power transmission system", Student Conference on Research and Development. (2002), pp. 265-268. 